\title{
Development of an on-the-fly data processing with information-lossless compression for CITIUS detectors at SPring-8
}

\author{
Toshiyuki Nishiyama Hiraki ${ }^{1}$, Toshinori Abe ${ }^{1,2}$, Mitsuhiro Yamaga ${ }^{1,2}$, Takashi Sugimoto ${ }^{1,2}$, Kyosuke Ozaki ${ }^{1}$, \\ Yoshiaki Honjo', Yasumasa Joti ${ }^{1,2}$, and Takaki Hatsui ${ }^{1}$ \\ ${ }^{1}$ RIKEN SPring-8 Center, \\ ${ }^{2}$ Japan Synchrotron Radiation Research Institute \\ toshiyuki.nishiyama@riken.jp
}

Diffraction-limited synchrotron radiation sources (DLSRs) using the advanced accelerator technologies deliver high-brilliance X-rays at high repetition rates. The DLSRs are expected to provide X-ray diffraction (XRD) measurements with benefits such as a reduction of the total time required for a complete scan and an improvement of temporal resolution. At the proposed SPring-8-II facility [1], one of the DLSRs, anticipated experiments using XRD techniques require X-ray imaging detectors with a frame rate over $10 \mathrm{kHz}$, high pixel count, a count rate over $100 \mathrm{Mcps} / \mathrm{pixel}$, and single-photon sensitivity. To meet these demands, we have been developing a highspeed X-ray imaging detector CITIUS (Charge Integration Type Imaging Unit with high-Speed extended-Dynamic-Range Detector) [2] for SPring-8 and SACLA. As for SPring-8, our first milestone is to install a 20M-pixel CITIUS detector in 2023. It has a frame rate of $17.4 \mathrm{kHz}$ and a raw data rate of $1.4 \mathrm{~TB} / \mathrm{s}$. Such a high raw data rate demands the careful design of the data handling scheme from the transfer, on-the-fly processing, storage, to post-analysis.

In this presentation, we describe our plan on the data acquisition and analysis scheme and the current status of the development. Our baseline implementation of the data-processing flow is composed of two steps. At the first step, detector images are processed by onthe-fly processing such as accumulation and a veto mechanism, which reduces the peak data-stream rate from $1.4 \mathrm{~TB} / \mathrm{s}$ to $\sim 400 \mathrm{~GB} / \mathrm{s}$. The processing algorithms are implemented onto custom PCB boards (Data Framing Board, DFB). Each DFB has three field-programmable gate arrays (FPGAs). Then generated processed data are transferred via PCI Express 3.0 bus to PC server memory. The second step is to compress the images by PC servers. We are investigating several information-lossless compression algorithms including the one presented in [3]. The peak data rate after the compression is further reduced to $\sim 10 \mathrm{~GB} / \mathrm{s}$. The compressed images are to be stored in cache storage with a capacity of about 4-day measurements. The cached data are transferred to the high-performance computing system for post-analysis, and long-term storage. We also present the results of the experiment using an X-ray photon correlation spectroscopy technique. We also present the infrastructure in detail to execute this flow.

[1] “SPring-8-II Conceptual Design Report” (Nov. 2014) http://rsc.riken.jp/eng/pdf/SPring-8-II.pdf.

[2] T. Hatsui, "New opportunities in photon science with high-speed X-ray imaging detector Citius, and associated data challenge", Presentation at the 2nd R-CCS International Symposium (2020)

[3] R. Roy et al., the proceedings of CCGrid2021, accepted.

Keywords: x-ray scattering; data transfer; computation 